\title{
Design methodology for a PM electrical variable transmission used in HEV
}

\author{
Florian Verbelen ${ }^{\mathrm{a}, \mathrm{b}}$, Hendrik Vansompel $\mathrm{l}^{\mathrm{a}, \mathrm{b}}$, Ahmed Abdallh $^{\mathrm{c}}$, Kurt Stockman ${ }^{\mathrm{a}, \mathrm{b}}$ and \\ Peter Sergeanta,b \\ aDepartment of Electrical Energy, Metals, Mechanical Constructions and Systems, Ghent University, \\ Belgium \\ ${ }^{b}$ Member of Energy Efficient Drive Trains partner of Flanders Make, www.eedt.ugent.be \\ cMotionS Core Lab at Flanders Make, Lommel, Belgium
}

\begin{abstract}
Designing a permanent magnet electrical variable transmission is a cumbersome task, regardless of the considered application. The main reason for this is the iterative design process using a computationally intensive finite element model calculations that is necessary to model its behaviour. This makes it difficult to study or visualize the impact of design changes on, for example, the fuel consumption or cost of a hybrid electrical vehicle. To solve this, electromagnetic scaling laws are used to set up a performance map of the entire system. This map is able to present the performance (i.e. fuel consumption, cost, maximum acceleration, etc.) as a function of an axial and radial scaling factor. The map thus displays the performance of a series of designs which enable the reader to select the optimal one in a graphical way. Furthermore, feasibility constraints such as maximum weight, are added. These constraints allow to reject designs but make it also possible to study the performance as a function of weight or material cost. This is particularly useful for manufacturers as it gives an idea of how their investment is translated into a reduction in fuel consumption.
\end{abstract}

\section{Introduction}

Vehicle manufacturers encounter an ever-growing pressure to reduce fuel consumption and emissions, because of increasingly stringent regulations. As a result, they are forced to innovate to find new solutions which can assist in reaching the climate goals. One of these solutions is the Hybrid Electrical Vehicle (HEV). The most important advantage of HEVs compared to classical vehicles is the higher efficiency which is due to the power split capabilities of the drivetrain. Important side-effects are friction and thus wear in the planetary gears that enable the power split but also the valuable space which is taken by the added electrical machines.

A solution that eliminates the planetary gearbox and the additional electrical machines is called the Electrical Variable Transmission (EVT) [1], [2]. The device consists of two rotors and one stator. In case of a HEV, the Internal Combustion Engine (ICE) is connected to the inner rotor and the wheels to the outer rotor. Both shafts are electrically coupled via inverters and the DC-bus, which can include a storage device. In an EVT, power can thus be transferred from the inner rotor to the outer rotor by means of an electric and/or electromagnetic path. Because the power is converted via two separate paths, the EVT can be considered as a power split device. Moreover, the EVT can also be used as an electromagnetic torque converter [3].

The behaviour of the EVT is typically studied based on time-consuming Finite Element (FE) calculations. Evaluating the effect of design changes of the EVT on the performance of a considered $\mathrm{HEV}$ is, therefore, a tedious task. However, due to developed scaling laws for the flux, torque and losses, an existing design of the EVT can be scaled in axial and radial directions which significantly reduces the computational effort to analyse a design [4]. In this present paper, the aforementioned method is used to analyse how the design of the EVT influences the energy efficiency of the complete drivetrain given a driving cycle and vehicle parameters.

The paper is structured as follows. In section 2, the topology, operating principle and control of the EVT is discussed. The scaling laws are introduced in section 3, while in section 4 the drivetrain and control of the considered HEV are elaborated. In section 5 the results are discussed. Finally in section 6 the conclusions of the research are formulated. 


\section{Permanent magnet Electrical Variable Transmission}

In this section, the topology of the considered EVT and the operating principle is explained as well as the control of the component.

\section{a) Topology}

A cross section of the considered EVT is shown in Fig. 1. The inner rotor and stator each consist of a distributed three-phase winding. The outer rotor is equipped with a single layer of rare-earth permanent magnets and a DC-field winding which are separated by a flux bridge. When the absolute value of the DC-field current increases, the magnetic state of the flux bridge located underneath the DC-field winding changes. The purpose of this DC-field winding is thus to modify the path of the permanent magnet flux which changes the stator flux linkage. The inner rotor field is almost not affected by the DC-field current since the magnets have a low recoil permeability of about 1.05 [5]. Using the DC-field current, it is thus possible to change the stator flux linkage, while maintaining the rotor flux linkage which is particularly useful in HEV applications [1], [5].

The shafts of the inner and outer rotor form the 2 mechanical ports while the windings of both the inner rotor and stator form the 2 electrical ports. To provide power to the inner rotor, slip rings are used. Some machine parameters are given in Table 1 for clarity.

Table 1: Machine parameters of the considered EVT.

\begin{tabular}{|l|c|c|c|}
\cline { 2 - 4 } \multicolumn{1}{c|}{} & Stator & Outer rotor & Inner rotor \\
\hline Rated mechanical power [kW] & - & 120 & 75 \\
\hline Maximal speed [rpm] & - & 4000 & 5000 \\
\hline Number of slots [-] & 48 & - & 48 \\
\hline Number of pole pairs [-] & 4 & 4 & 4 \\
\hline Outer radius [mm] & 175 & 123.5 & 102 \\
\hline Inner radius [mm] & 124.5 & 103 & 57 \\
\hline PM thickness [mm] & - & 5 & - \\
\hline Active axial length [mm] & 87 & 87 & 87 \\
\hline Width flux bridge [mm] & - & 5 & - \\
\hline
\end{tabular}

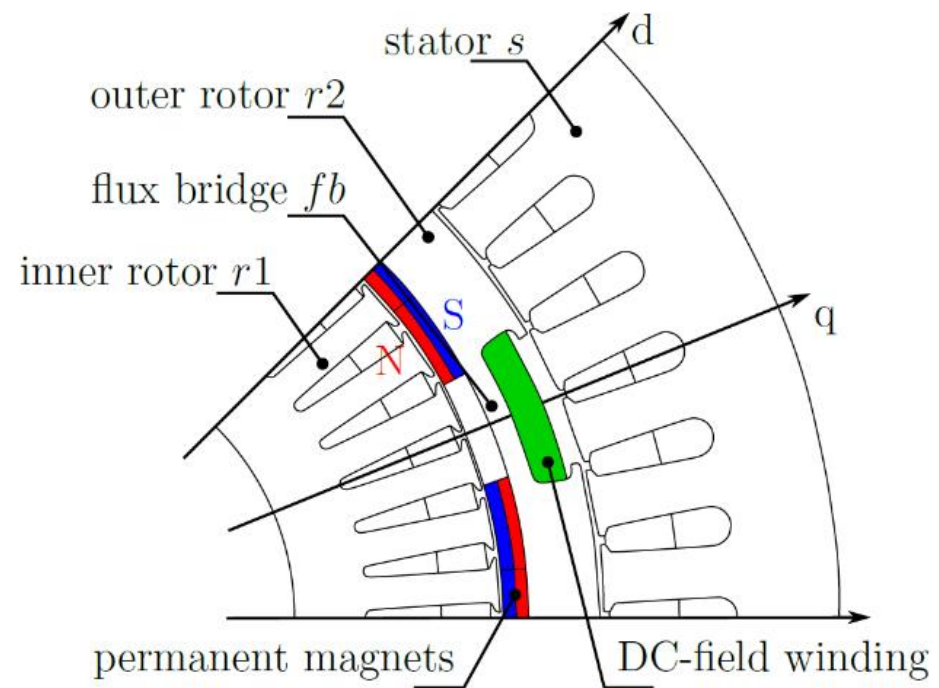

Figure 1: Cross section of the considered PM EVT with a hybrid excitation.

b) Operating principle 
One of the main advantages of the EVT is that there is no mechanical connection between the inner rotor and the outer rotor. Moreover, in a HEV, the EVT decouples the ICE (connected to the inner rotor) and the wheels (connected to the outer rotor) in terms of speed and torque (see Fig. 4). This makes it possible to operate the ICE independently from the wheels. Consequently, the ICE can be operated in its operating point of maximum efficiency at all times. Why the EVT is decoupled in terms of speed is rather trivial: there is no mechanical link between both shafts and as a consequence they can rotate at different speed. To explain why the EVT also decouples input and output in terms of torque, the EVT is first considered without stator. Suppose that the ICE is operating in the following operating point: $T_{I C E}$ and $N_{I C E}$. Then the inner rotor should, in steady state, produce a torque equal to the opposite of the ICE torque in order to maintain the speed:

$$
T_{r 1}=-T_{I C E}
$$

In order to produce this torque, currents need to be sent through the windings of the inner rotor:

$$
T_{r 1}=\frac{3}{2} N_{p}\left(\psi_{r 1 q} I_{r 1 d}-\psi_{r 1 d} I_{r 1 q}\right)
$$

in which flux $\psi$ and current $I$ are related to the $d$ - $q$ reference frame (related to the outer rotor) and $N_{p}$ is the number of pole pairs. Due to action-reaction, the electromagnetic torque on the outer rotor will produce the opposite torque. Therefore, the following is true:

$$
T_{r 2}=T_{I C E}
$$

The torque at the wheels is thus the same as the torque generated by the ICE. Note that the following is of course only true in lossless conditions. To be able to modify the torque at the outer rotor, the stator is added. By sending currents through the stator windings, an electromagnetic torque is added to the outer rotor. Since the sum of the torque on all components needs to be 0 , the torque on the outer rotor $T_{r 2}$ can be determined by:

$$
T_{r 2}=-T_{s}-T_{r 1}=-T_{s}+T_{I C E}
$$

in which the stator torque $T_{s}$ is calculated as:

$$
T_{s}=\frac{3}{2} N_{p}\left(\psi_{s q} I_{s d}-\psi_{s d} I_{s q}\right)
$$

The fluxes (stator flux in the d-axis $\psi_{s d}$, stator flux in the $q$-axis $\psi_{s q}$, inner rotor flux in the $d$-axis $\psi_{r 1 d}$ and inner rotor flux in the $q$-axis $\psi_{r 1 q}$ ) in (2) and (5) depend on 5 independent current setpoints: stator current in the $d$-axis $I_{s d}$, stator current in the $q$-axis $I_{s q}$, current in the DC-field winding of the outer rotor $I_{r 2 d}$, inner rotor current in the $d$-axis $I_{r 1 d}$ and inner rotor current in the $q$-axis $I_{r 1 q}$. The relation between flux and current is determined based on FE calculations. The FE model, which forms the backbone of the EVT model, has been validated on a prototype [5].

\section{c) Optimal control}

Based on (2) and (5) and knowing that the flux terms in these equations depend on 5 current setpoints, it is possible to deduce that an infinite number of current combinations will result in the same torque. This can also be seen in Fig. 2, which shows that a stator torque of, for example, $100 \mathrm{Nm}$ can be obtained with various current combinations. Of course, only one of these combinations will result in the lowest losses. 


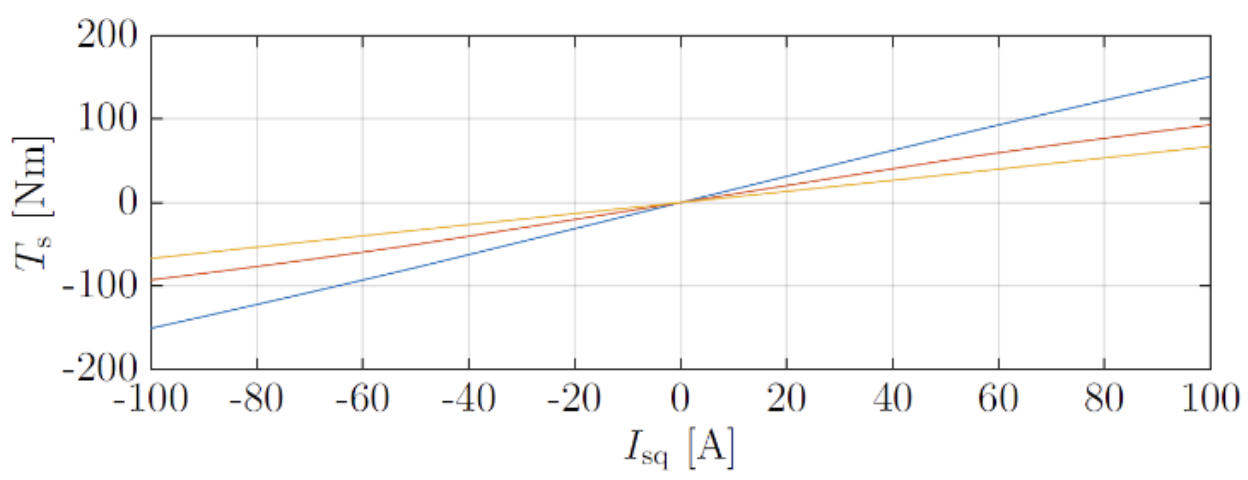

Figure 2: Stator torque as function of stator current in the q-axis for varying $D C$-field current. Blue: $I_{r 2 d}=-10 A$, red: $I_{r 2 d}=-5 A$ and orange: $I_{r 2 d}=-0 A$. All other currents are set to 0 .

The optimization routine which is written to find the optimal currents, given a specific operating point, can be summarized as follows [6]:

Find:

$$
I^{*}=\left[I_{s q}^{*}, I_{s d}^{*}, i_{r 2 d}^{*}, I_{r 1 q}^{*}, I_{r 1 d}^{*}\right] \in \mathbb{R}^{5}
$$

with:

$$
I^{*}=\arg \min \left(P_{\text {loss }}\left(I, N_{r 1}, N_{r 2}\right)\right)
$$

subjected to:

$$
T_{s}\left(I^{*}\right)=T_{s, \text { set }} \& T_{r 1}\left(I^{*}\right)=T_{r 1, \text { set }}
$$

The generated torque is calculated based on (2) and (5), $P_{\text {loss }}$ is equal to the sum of iron and copper losses (mechanical losses are neglected in this optimization). As solving this routine takes some time, the optimization is carried out offline after which the results are stored in a Look-Up Table (LUT) which is consulted during operation. The LUT thus basically yields the optimal current setpoints given an operating point defined by a stator $T_{S}$ and inner rotor $T_{r 1}$ torque setpoint and inner and outer rotor speed denoted by $N_{r 1}$ and $N_{r 2}$ respectively.

\section{Scaling laws}

As mentioned in the introduction, analysis of the performance of the EVT depends on the numerous FE calculations which are necessary to define the model. Therefore, the computational burden of studying the impact of design changes is high. Scaling laws, however, can be used to obtain a new design in a matter of minutes compared to several days if FE calculations are used [4]. One way of reducing the computational effort is thus to use scaling laws.

Scaling laws allow to scale an EVT of which all current to flux relations are known in axial and radial directions. During axial scaling, the axial core length is changed while preserving the lamination crosssection. The axial scaling factor $K_{A}$ can therefore be calculated as:

$$
K_{A}=\frac{l_{a}}{l_{a, 0}}
$$

in which $l_{a, 0}$ is the axial length of the reference EVT and $l_{a}$ is the length of the scaled design. Radial scaling considers a proportional change of all dimensions of the cross-section. The radial scaling factor is thus equal to: 


$$
K_{R}=\frac{D}{D_{0}}
$$

in which $D_{0}$ is the diameter of the reference EVT and $D$ is the diameter of the scaled design.

In the following sections, the scaling laws as derived and validated in [4] are mentioned for clarity.

\section{a) Scalable machine properties}

The most important properties for this analysis are current, flux, torque, copper and iron losses. However, scaling laws can be derived for all machine properties including inductance, voltage, etc. [7]. Based on the Poisson's equation it is possible to derive that the current needs to scale as follows [4], [7]:

$$
I=K_{R} I_{0}
$$

with $I_{0}$ the current which flows in the reference machine and $I$ the current in the scaled design. Note that both $I_{0}$ and $I$ are arrays which contain 5 current setpoints $\left(I_{s q}^{*}, I_{s d}^{*}, i_{r 2 d}^{*}, I_{r 1 q}^{*}, I_{r 1 d}^{*}\right)$. If the relation in (11) is met than the flux $\psi$ and torque $T$ will scale as:

$$
\begin{aligned}
& \psi=K_{A} K_{R} \psi_{0}\left(I_{0}\right)=K_{A} K_{R} \psi\left(\frac{I}{K_{R}}\right) \\
& T=K_{A} K_{R}^{2} T_{0}\left(I_{0}\right)=K_{A} K_{R}^{2} T_{0}\left(\frac{I}{K_{R}}\right)
\end{aligned}
$$

The copper losses are calculated based on the currents flowing through the scaled EVT:

$$
P_{c u}=\frac{3}{2} R_{s}\left(I_{s d}^{2}+I_{s q}^{2}\right)+R_{r 2} i_{r 2 d}^{2}+\frac{3}{2} R_{r 1}\left(I_{r 1 d}^{2}+I_{r 1 q}^{2}\right)
$$

in which the resistance of the inner rotor, outer rotor and stator scale according to the following equation:

$$
R=\frac{1}{K_{R}^{2}}\left(K_{A} R_{c o, 0}+K_{R} R_{e w, 0}\right)
$$

in which $R_{c o, 0}$ is the resistance of the reference EVT in the core region, while the resistance of the end winding is denoted by $R_{e w, 0}$.

Finally, the iron losses [W/kg] are determined assuming a sinusoidal air gap induction waveform, using Bertotti's model [8]:

$$
P_{f e}=a \hat{B}^{\alpha} f+b \hat{B}^{2} f^{2}+c \hat{B} f(\sqrt{1+d \hat{B} f}-1)
$$

with a, b, c, d and $\alpha$ magnetic core material parameters, $\hat{B}$ the amplitude of the magnetic flux density and $f$ the frequency. The constants have been determined based on measurements.

\section{b) Scalable control}

In the previous section, it is shown how the EVT is scaled. However, setting up the optimal control is also a cumbersome task. Defining scaling laws for the optimal control (selection of the optimal currents, 
given the operating point), is thus also important. In [4], it is shown that this scalable control can be obtained by scaling the torque $\left(T_{r 1}^{s}\right.$ and $T_{s}^{s}$, see Fig. 3$)$ which is used as input for the LUT containing the optimal currents $\left(I_{s d}^{s}, I_{s q}^{s}, \ldots\right)$. As these currents are obtained based on the LUT of the reference machine, these currents need to be scaled back to the scaled device. Therefore, the final step is to scale the currents with $K_{R}$ according to (11).

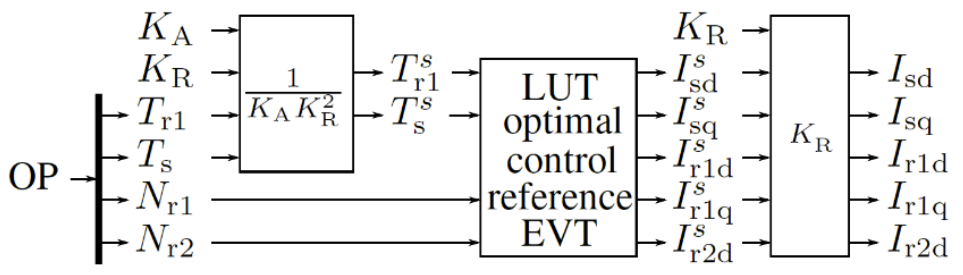

Figure 3: Schematic of the scalable optimal control.

\section{EVT based HEV}

In this paper, the main application in which an EVT can be used is a HEV (see Fig. 4). The HEV that is considered, both the drivetrain and its optimal control, are presented in the following sections. 


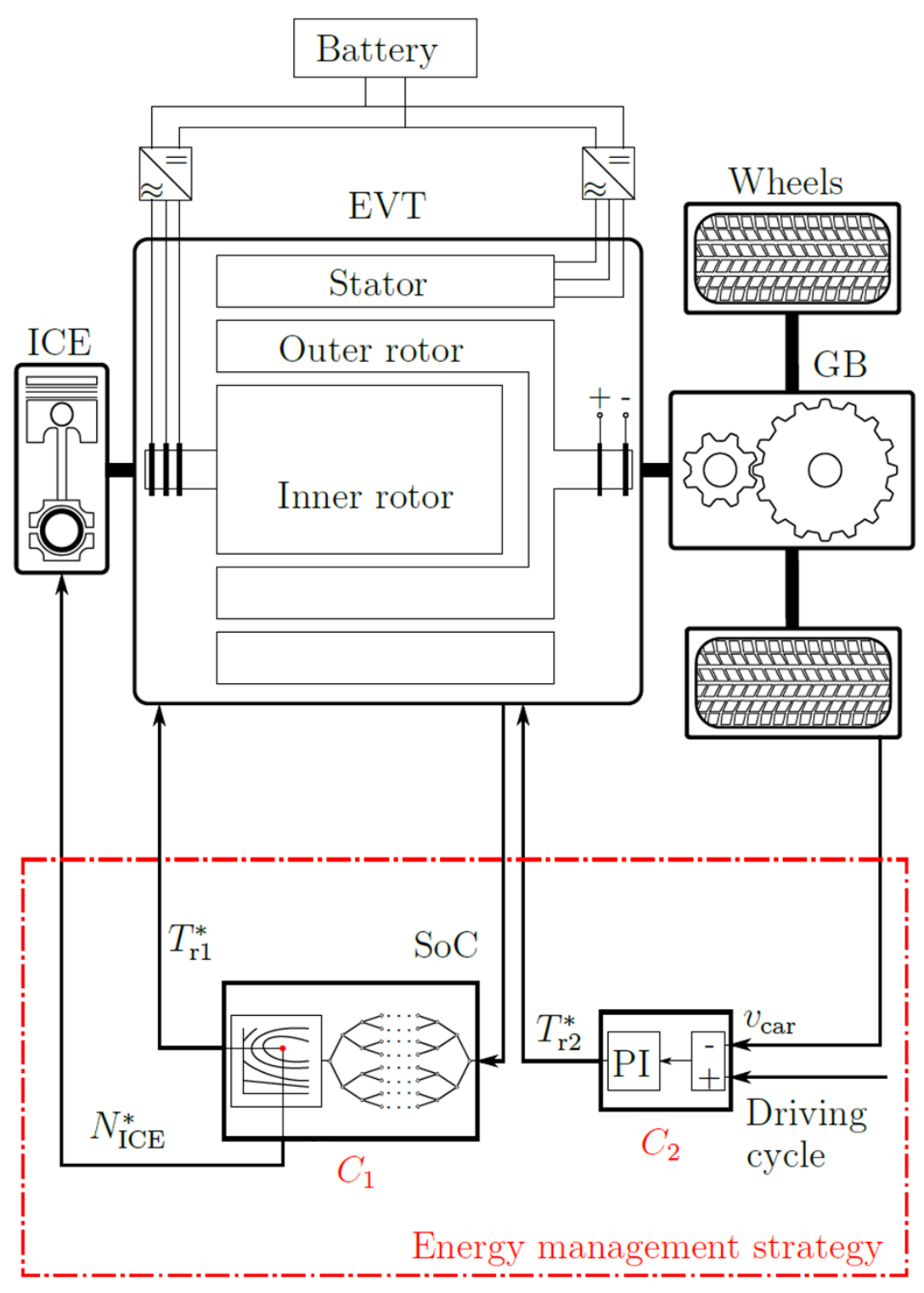

Figure 4: Schematic of the EVT based HEV drivetrain and simplified representation of the energy management strategy. More information concerning the energy management strategy (controllers $C_{1}$ and $C_{2}$ ) is given in section $3 \mathrm{~b}$.

\section{a) Vehicle}

Fig. 4 gives a schematic overview of the complete HEV drivetrain. In accordance with previously published literature [6], [9], the inner rotor of the EVT is connected to the ICE while the outer rotor is connected, via a gearbox, to the wheels of the vehicle. The purpose of this differential gearbox is to deliver torque to both wheels. The used ICE is a turbocharged gasoline engine (direct injection) running on a Miller cycle. The fuel map of the ICE will be used to calculate the fuel consumption for various design sizes of the EVT. The fuel consumption map of the ICE is presented in Fig. 5. 


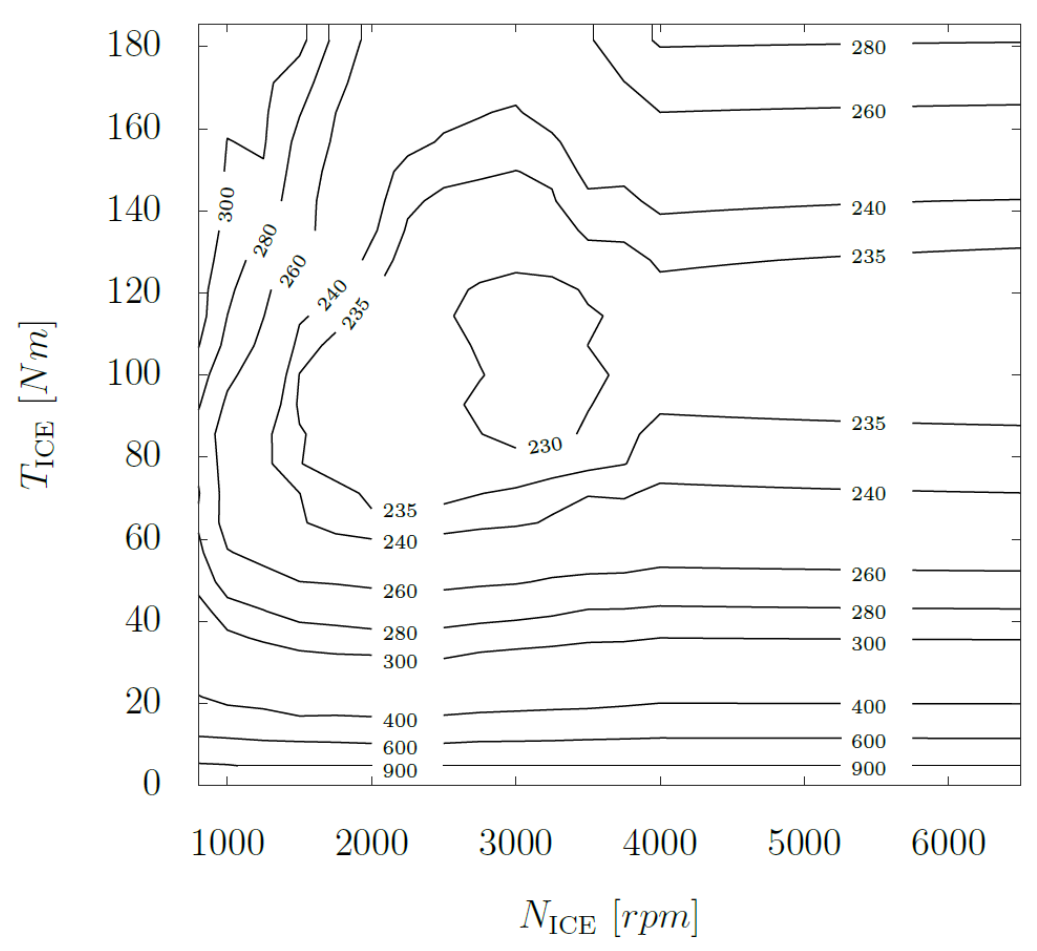

Figure 5: Fuel consumption [g/kWh] of a turbocharged gasoline engine (direct injection) running on a Miller cycle.

The behaviour of the vehicle is studied based on a 1-DOF model:

$$
v_{c a r}=\int \frac{\frac{T_{w}}{r_{w}}-m f g-\frac{1}{2} v_{c a r}^{2} \rho A_{f} c_{d}}{m}
$$

in which $v_{c a r}$ is the speed of the vehicle, $T_{w}$ is the torque at the wheels, $r_{w}$ is the wheel radius, $m$ is the mass of the vehicle (including passengers), $g$ is the gravitational constant, $f$ is the rolling resistance coefficient, $\rho$ is the density of air, $A_{f}$ is the frontal cross sectional area and $c_{d}$ is the aerodynamic drag coefficient. The main parameters of this vehicle model can be found in Table 2.

Table 2: Vehicle parameters.

\begin{tabular}{|l|l|}
\hline Mass $m[\mathrm{~kg}]$ & 1400 \\
\hline Aerodynamic drag coefficient $c_{d}[-]$ & 0.28 \\
\hline Frontal cross sectional area $A_{f}\left[\mathrm{~m}^{2}\right]$ & 1.5 \\
\hline Rolling resistance coefficient $f[-]$ & 0.009 \\
\hline Wheel radius $r_{w}[\mathrm{~m}]$ & 0.3 \\
\hline Battery capacity $C_{b a t}[\mathrm{kWh}]$ & 1.8 \\
\hline
\end{tabular}

To transfer the torque of the outer rotor to the wheels, a differential gearbox is used. The speed ratio of this gearbox, $\tau$, describes the relation between the wheel speed $N_{w}$ and the speed of the outer rotor $N_{r 2}$ as follows:

$$
\tau=\frac{N_{w}}{N_{r 2}}
$$

If $\tau$ is smaller than 1 , the outer rotor will rotate faster than the wheels. The wheel torque $T_{w}$, as mentioned in (9) is related to the outer rotor torque $T_{r 2}$ of the EVT by the following equation:

$$
T_{w}=\frac{T_{r 2} \eta}{\tau}
$$


in which $\eta$ is the efficiency of the gearbox. Note that during braking, the efficiency term goes to the denumerator as power flows from the wheels to the inner rotor. Both the wheel speed and the wheel torque are imposed by the driving cycle. The wheel speed is directly related to the vehicle speed and the wheel torque is related to the acceleration and speed of the vehicle (due to the speed dependency of for example the aerodynamic drag) but also the road gradient (ignored in (9)). The selected driving cycle for this analysis is the WLTC (see Fig. 6).

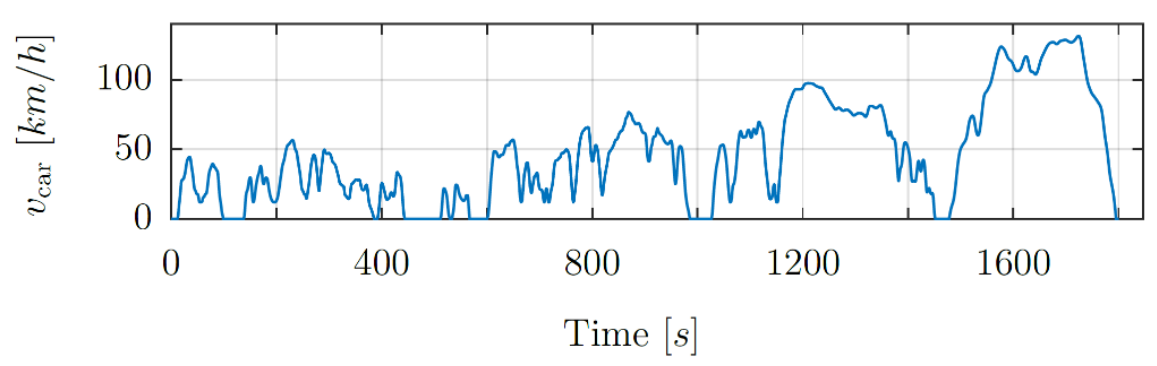

Figure 6: Imposed vehicle speed [km/h] as function of time for the WLTC.

Finally, the battery is considered as an ideal storage device (no losses) as the main goal of this paper is to show how the scaling laws can be applied. The State of Charge (SoC) of the battery is calculated as:

$$
S o C=S o C_{0}-\frac{\int P_{b a t}}{3600 C_{b a t}}
$$

with $S o C_{0}$ the state of charge at the beginning of the cycle and $P_{b a t}$ the battery power. The battery power is calculated as the sum of the electrical power of the stator $P_{e, s}$ and inner rotor $P_{e, r 1}$ (including losses):

$$
P_{b a t}=P_{e, s}+P_{e, r 1}
$$

\section{b) Control}

The objective of this paper is to find the optimal size of the EVT in an EVT based HEV. The fuel consumption of the vehicle is chosen to quantify how optimal a considered drivetrain is. The chosen operating points of the ICE ( $C_{1}$ see Fig. 4 ), however, have a significant impact on the fuel consumption. To exclude the impact of controller $C_{1}$, Dynamic Programming (DP) is applied in order to find the optimal control regardless of the drivetrain configuration.

DP has been used successfully to optimize the fuel consumption of a HEV taking into account several constraints such as the battery SoC [10]-[12]. Hence, the SoC at the beginning and end of the driving cycle should be the same. In this paper, the open source toolbox provided by Sundström and Guzella had been used as framework for the DP structure [13].

Besides controller 1 of which the output is defined based on DP, a second controller ( $C_{2}$ see Fig. 4 ), is used to adapt the outer rotor torque in such a way that the imposed speed, defined based on the driving cycle, is achieved with a maximum error of $1 \mathrm{~km} / \mathrm{h}$ [14].

\section{Results}

The objective is to optimize the drivetrain as depicted in Fig. 4. This comes down to optimizing the size of the EVT and the end gearbox. For the EVT, a range of possible designs defined by an axial and radial scaling factor varying from 0.8 to 1.2 are defined. For each design, the gear ratio is searched for 
that minimizes the fuel consumption. The fuel consumption itself is optimized based on DP. The DP algorithm is thus solved for every considered design.

The results (fuel consumption) are stored in a performance map which shows how the fuel consumption varies with the scaling factor, see Fig. 7. However, this performance could also be quantified by, for example, the maximum acceleration.

The performance map shows that, the bigger the EVT is chosen, the lower the fuel consumption becomes. This is not a surprise as bigger electrical machines tend to have lower losses due to the lower currents which are necessary to generate the same torque compared to a smaller machine. However, bigger machines come with a higher material cost. Furthermore, space is limited under the vehicle frame which limits the size of the EVT. Besides the fuel consumption, Fig. 7 also shows the chosen gear ratio for the end gearbox. The figure shows that the variation in optimal gear is rather small: it varies between 0.2 and 0.3 .

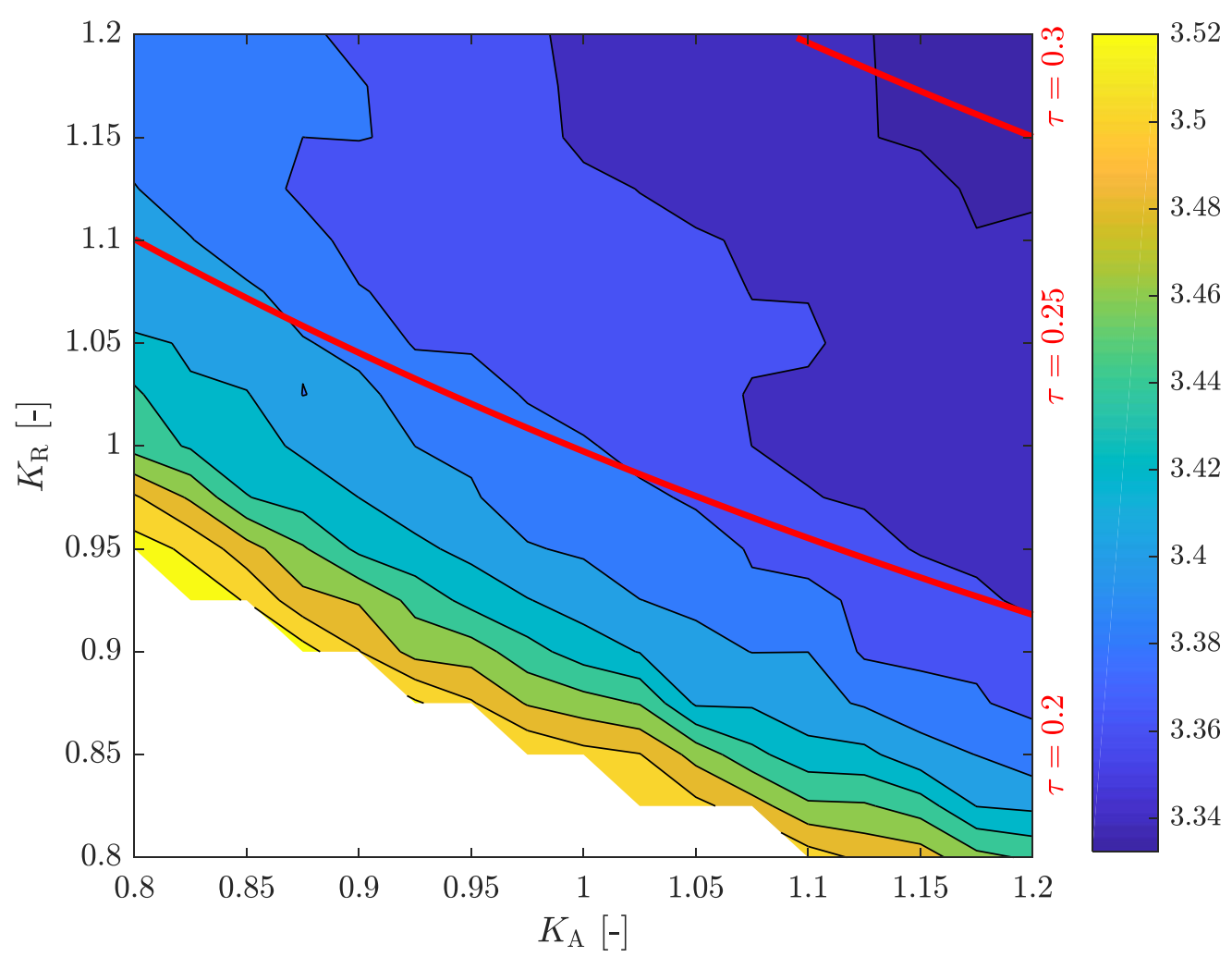

Figure 7: Performance map showing the fuel consumption of the HEV during the WLTC for varying axial and radial scaling factor. The drivetrains that use an EVT, defined by a $K_{A}$ and $K_{R}$, situated below the lowest red line use an end gearbox with a speed ratio $\tau$ equal to 0.2. Between the 2 red lines, $\tau$ equals 0.25 and above the highest red line $\tau$ equals 0.3 .

To make the results in Fig. 7 a bit more tangible, the scaling factors are translated to actual dimensions (see Fig. 8). Note that the scaling factors are related to the active part of the EVT, while the total length and diameter cover also the housing. In the radial direction, the difference between the active part and housing, that includes a water jacket cooling, is rather small. However, in the axial direction, the active part is combined with a set of AC slip rings for the inner rotor and a set of DC slip rings for the DC-field winding of the outer rotor. This creates a rather significant difference between the active part and the total length of the EVT. Consequently, the variation of the axial scaling factor between 0.8 and 1.2 results only in a variation in total length of about $35 \mathrm{~mm}$. 


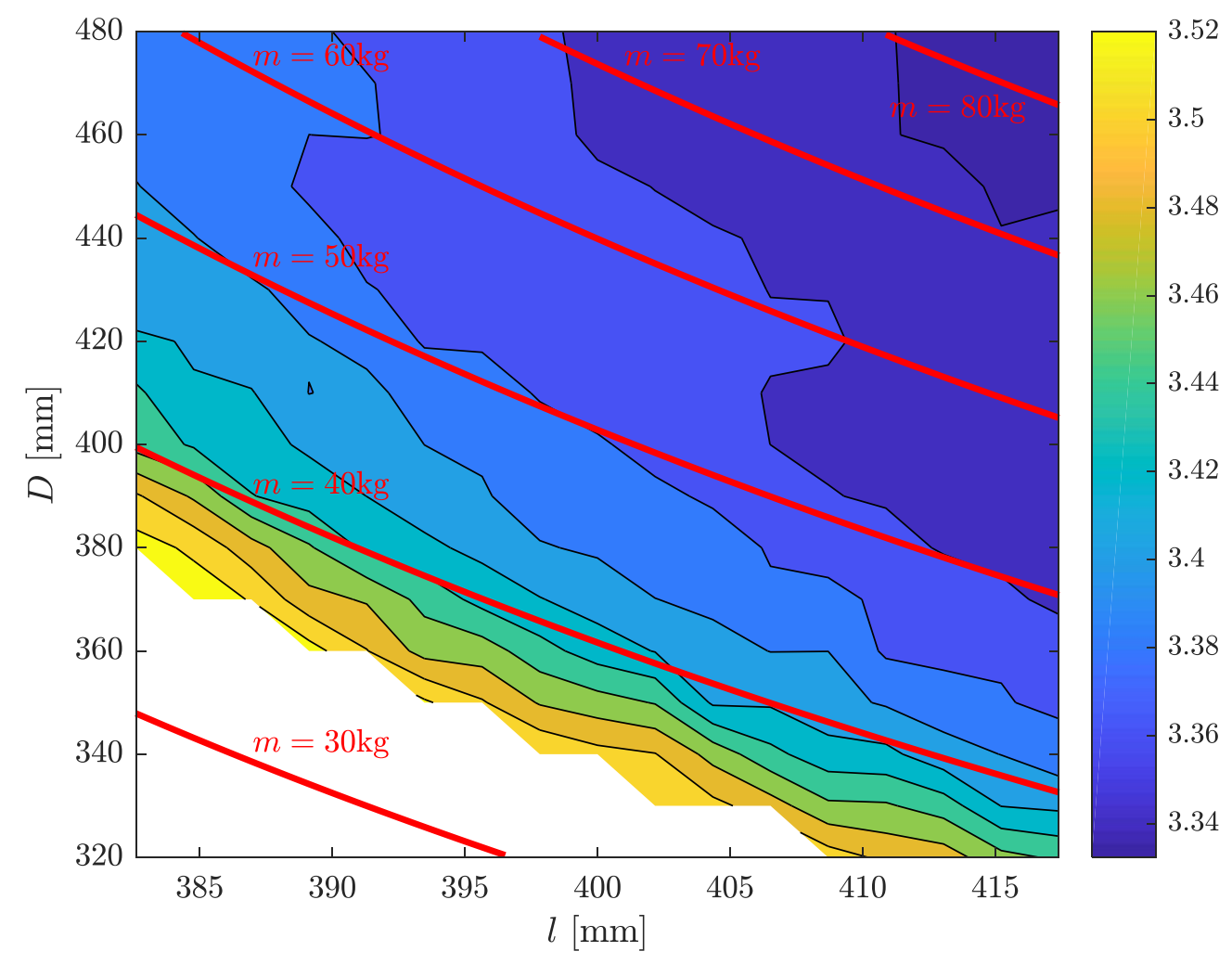

Figure 8: Performance map showing the fuel consumption of the HEV during the WLTC for varying length and diameter of the housing. The red lines resemble designs with a constant mass.

Fig. 8 also shows lines of constant weight which make it possible to approximate the minimal weight (i.e. size) of the EVT for the used load cycle. As already stated, bigger and thus heavier machines result in lower losses. To quantify this, Fig. 8 can be used to calculate the fuel consumption for a given maximum weight. This comes down to finding the optimal design for a given mass. The results are presented in Fig. 9. This figure is particularly useful for manufacturers because it gives an idea of how the sizing influences the fuel consumption. It shows that increasing the weight beyond $50 \mathrm{~kg}$ results only in marginal gains in terms of overall fuel consumption. Although it is perfectly feasible to reduce the weight to $40 \mathrm{~kg}$, Fig. 9 clearly demonstrates that minimizing the EVT even further has a highly negative impact on the fuel consumption.

Fig. 10 shows the same information as in Fig. 9 but now as function of material cost. This figure, immediately shows, how high the gain is of resizing the EVT. If the design cost is for example estimated at 400 euro, than an increase in material cost of 100 euros will result in a decrease in fuel consumption of about $0.071 / 100 \mathrm{~km}$. However, a further increase of the budget with 100 euro will only decrease the fuel consumption by $0.031 / 100 \mathrm{~km}$. Again, if the manufacturer wants a product with a material cost below 400 euro, that is perfectly possible but at the cost of a higher fuel consumption per $100 \mathrm{~km}$. The vehicle manufactures can use this information to draw a conclusion about the sizing of EVT considering the trade-off between the initial cost and the running cost over the vehicle lifetime. 


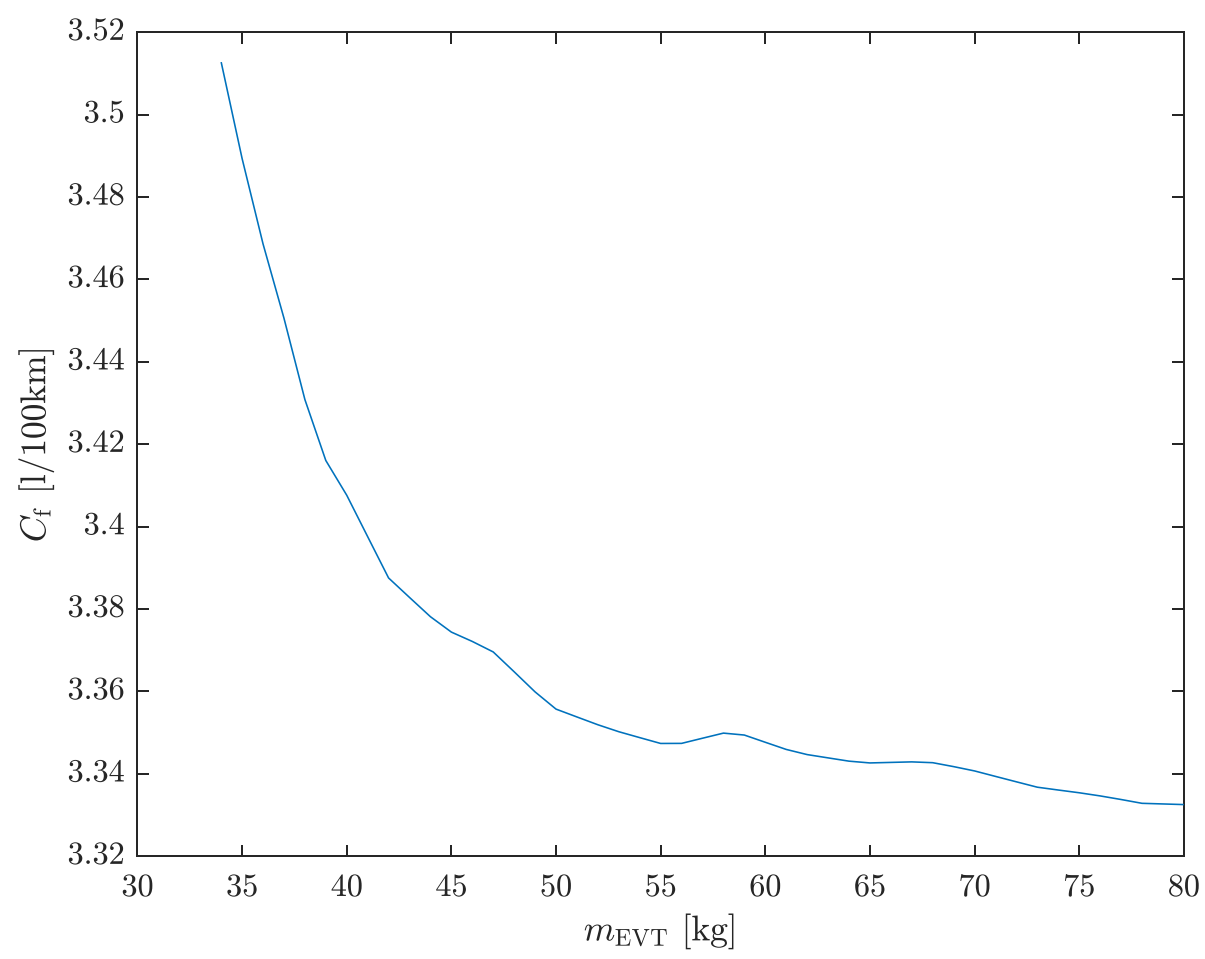

Figure 9: Fuel consumption in $\mathrm{I} / 100 \mathrm{~km}$ for the WLTC as function of the weight of the considered EVT design.

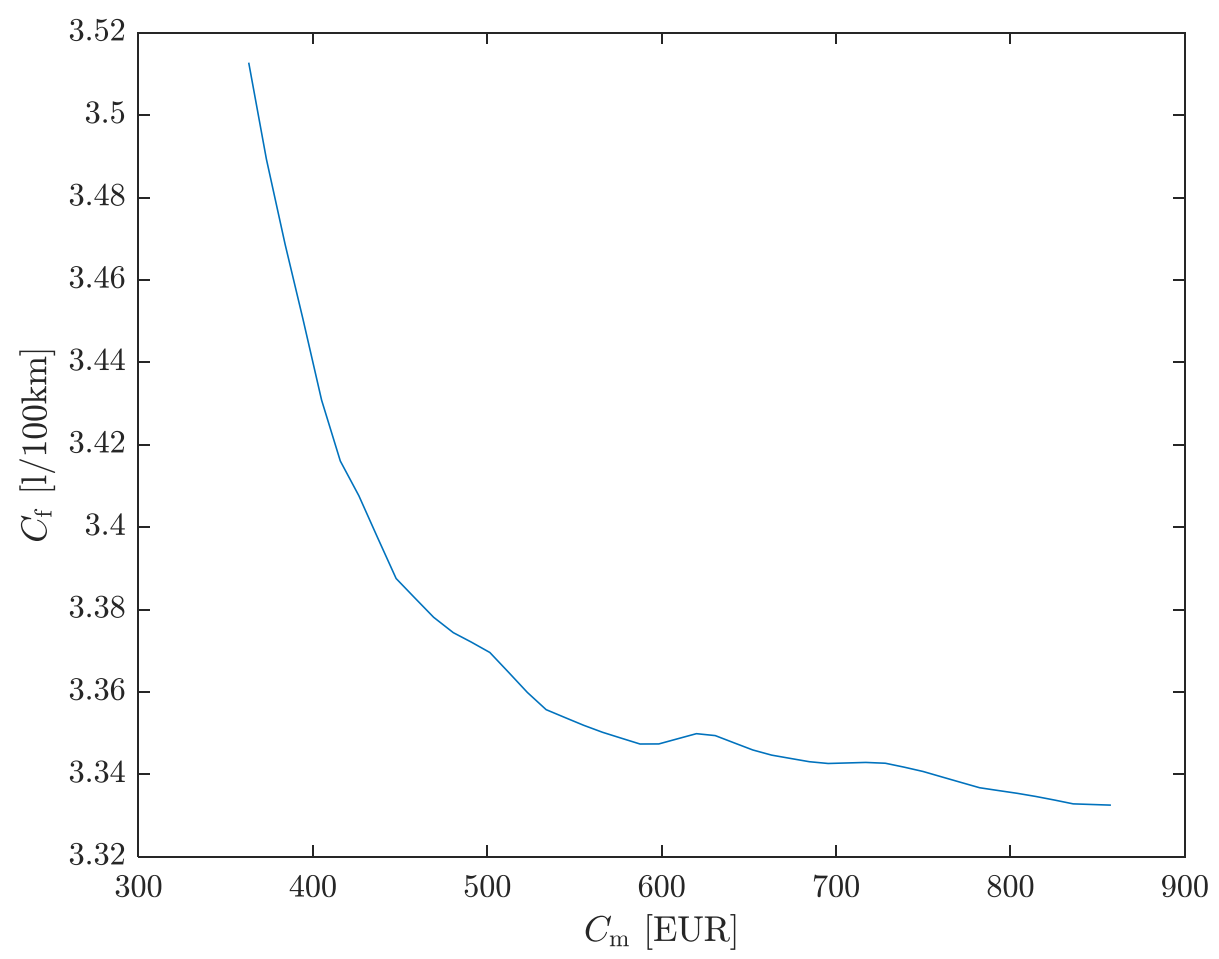

Figure 10: Fuel consumption in $1 / 100 \mathrm{~km}$ for the WLTC as function of the material cost of the considered EVT design.

\section{Conclusion}

In this paper, a method is described to aid in the design of an electrical variable transmission based on scaling laws. The principle of a performance map is used to analyze how well the considered design, defined by an axial and radial scaling factor, performs given a load cycle. The parameter used in this 
paper to define the performance is the fuel consumption. The feasibility of the design in terms of dimensions can be deduced easily based on the performance map by translating scaling factors to actual dimensions. Furthermore, constraints such as maximum weight can be added as well. Finally it is demonstrated that performance in terms of fuel consumption can be calculated in terms of material cost. This is particularly useful for manufacturers as it gives an idea of how their investment is translated in fuel consumption and consequently to meet the global emission requirements.

\section{Acknowledgement}

This work was carried out for the EMTechno project (project ID: IWT150513) supported by VLAIO and Flanders Make, the strategic research centre for the manufacturing industry in Belgium.

\section{References}

[1] M. Hoeijmakers, "Electromechanical converter, Patent US 7164 219," 2007.

[2] M. Hoeijmakers and J. Ferreira, "The electric variable transmission," IEEE Trans. Ind. Appl., vol. 42, no. 4, pp. 1092-1100, 2006.

[3] S. Vanhee, "Concept Study of an Electrical Variable Transmission for Off-Highway Applications," Master thesis, Ghent University, Belgium, 2015.

[4] F. Verbelen, A. Abdallh, H. Vansompel, K. Stockman, and P. Sergeant, "Sizing Methodology based on Scaling Laws for a Permanent Magnet Electrical Variable Transmission," IEEE Trans. Ind. Electron., 2019.

[5] J. Druant, H. Vansompel, F. De Belie, J. Melkebeek, and P. Sergeant, "Torque Analysis on a Double Rotor Electrical Variable Transmission With Hybrid Excitation," IEEE Trans. Ind. Electron., vol. 64, no. 1, pp. 60-68, 2017.

[6] J. Druant, H. Vansompel, F. De Belie, and P. Sergeant, "Optimal Control for a Hybrid Excited Dual Mechanical Port Electric Machine," IEEE Trans. Energy Convers., vol. 32, no. 2, pp. 599607, 2017.

[7] S. Stipetic, D. Zarko, and M. Popescu, "Ultra-fast axial and radial scaling of synchronous permanent magnet machines," IET Electr. Power Appl., vol. 10, no. 7, pp. 658-666, 2016.

[8] G. Bertotti, Hysteresis in magnetism: for physicists, materials scientists, and engineers. Academic Press, 1998.

[9] J. Druant, H. Vansompel, F. De Belie, and P. Sergeant, "Efficiency of a CVT Operated EVT Experimentally Evaluated against Half-Toroidal and Push-belt CVTs," IEEE Trans. Ind. Electron., vol. 65, no. 4, pp. 3095-3103, 2018.

[10] E. Vinot, R. Trigui, Y. Cheng, C. Espanet, A. Bouscayrol, and V. Reinbold, "Improvement of an EVT-Based HEV Using Dynamic Programming," IEEE Trans. Veh. Technol., vol. 63, no. 1, pp. 40-50, 2014.

[11] Z. Chen, C. C. Mi, and J. Xu, "Energy Management for a Power-Split Plug-in Hybrid Electric Vehicle Based on Dynamic Programming and Neural Networks," IEEE Trans. Veh. Technol., vol. 63, no. 4, pp. 1567-1580, 2014.

[12] E. Vinot, "Comparison of different power-split hybrid architectures using a global optimisation design method," Int. J. Electr. Hybrid Veh., vol. 8, no. 3, pp. 225-241, 2016.

[13] O. Sundström and L. Guzzella, "A Generic Dynamic Programming Matlab Function," in IEEE Multi-conference on Systems and Control, 2009, pp. 1625-1630.

[14] UN, "Proposal for a new UN Global Technical Regulation on Worldwide harmonized Light vehicles Test Procedures (WLTP)," Economic and Social Council, p. 235, 2013. 